\title{
Bienestar Psicológico, Apoyo Social Percibido y Percepción de Salud en Adultos Mayores
}

\author{
Psychological Well-Being, Perceived Social Support and Health \\ Perception Among Older Adults
}

\author{
Flavia Vivaldi \\ $\&$ \\ Enrique Barra \\ Universidad de Concepción, Chile
}

(Rec: 22 de septiembre de 2010 / Acep: 20 de marzo de 2012)

\begin{abstract}
Resumen
Este estudio tuvo como objetivo examinar las relaciones entre el bienestar psicológico, el apoyo social percibido y la percepción de salud en adultos mayores. La muestra estuvo constituida por 250 personas, 146 mujeres y 104 hombres, con edades entre 60 y 87 años (promedio de 70,8 años) de la ciudad de Concepción (Chile). Los resultados mostraron que el bienestar psicológico presentaba mayores relaciones con el apoyo social percibido que con la percepción de salud, que los hombres informaron mayor bienestar psicológico y mejor percepción de salud que las mujeres y que las personas casadas o con pareja estable informaron mayor bienestar psicológico y mayor apoyo social percibido que aquellas sin pareja estable. Se proponen diversos factores explicativos para los resultados obtenidos.
\end{abstract}

Palabras clave: Bienestar psicológico, apoyo social, adultos mayores

\begin{abstract}
This study examined the relationship between psychological well-being, perceived social support and health perception in older adults. The participants were 250 males and females with mean age of 71 years, from Concepción (Chile). The results showed that psychological well-being had a higher relationship with perceived social support than with health perception, that males reported higher psychological well-being and better health perception than females, and that married participants reported higher psychological wellbeing and higher perceived social support than participants without a partner. Diverse explanatory factors for obtained results are proposed.
\end{abstract}

Keywords: Psychological well-being, social support, older adults

\section{Introducción}

Uno de los cambios demográficos más significativos del último tiempo es el incremento en la proporción de adultos mayores en la población general, relacionado con el aumento en la expectativa de vida de este grupo etario y el descenso en la tasa de natalidad (Mella, González, D' Appolonio, Maldonado, Fuenzalida \& Díaz, 2004). En Chile, en el año 2001, los adultos mayores conformaban un $10,6 \%$ de la población y se espera que en el año 2025 alcancen un $19 \%$ de la población total del país (Sanhueza, Castro \& Merino, 2005).
El aumento en la expectativa de vida implica el desafío de adaptarse a los diversos cambios propios de la edad avanzada y enfrentar experiencias de pérdidas y amenazas al bienestar personal (Mella et al., 2004; Molina \& Meléndez, 2006; Prieto et al., 2008). Entre tales experiencias se encuentran la viudez, la jubilación, cambios de residencia, menor participación en la sociedad y mayor vulnerabilidad ante acontecimientos inesperados (Rioseco, Quezada, Ducci \& Torres, 2008). Las experiencias que el individuo adquiere a lo largo de su vida pueden hacer que el adulto mayor se sienta realizado o en el otro extremo desesperanzado y marginado del resto. Si bien los estereotipos peyorativos

Correspondencia: La correspondencia relacionada con este artículo debe ser enviada a Flavia Vivaldi Calbacho. Departamento de Psicología, Universidad de Concepción, Casilla 160-C, Concepción, Chile. E-mail: fvivaldi@udec.cl 
de la vejez aún persisten, han ido disminuyendo en los últimos años para privilegiar una concepción más positiva del envejecimiento exitoso (Aguerre \& Bouffard, 2008; Boone \& Wink, 2006). El envejecimiento exitoso implica que el individuo sea capaz de integrarse y desenvolverse de manera eficaz tanto en su familia como en la sociedad, superando las barreras culturales que le impidan desarrollar todo su potencial y mantener un nivel adecuado de bienestar personal.

El presente estudio estuvo destinado a examinar el bienestar psicológico de un grupo de adultos mayores de la ciudad de Concepción y sus relaciones con el apoyo social percibido y la autopercepción del estado de salud, junto con examinar posibles diferencias de género y de estado civil en las variables examinadas.

En la investigación sobre el bienestar es posible distinguir dos perspectivas generales. La primera, denominada tradición hedónica, estudia el bienestar subjetivo relacionado con la evaluación global que realiza el individuo de las situaciones tanto placenteras como las que no lo son. Esta perspectiva se relaciona con aspectos como la satisfacción vital, la felicidad y los afectos positivos (Díaz et al., 2006; García \& González, 2000; Pinguart \& Sorensen, 2001; Tomás, Meléndez \& Navarro, 2008). Mientras que la segunda perspectiva, llamada tradición eudaimónica, estudia el bienestar psicológico considerado como indicador de un funcionamiento positivo en el individuo, que posibilita el desarrollo de sus capacidades y por tanto su crecimiento personal.

La perspectiva eudaimónica ha sido sistematizada por Ryff en su modelo multidimensional de bienestar psicológico conformado por seis dimensiones (Ryff \& Singer, 2008), que incluyen la evaluación positiva de uno mismo y la propia vida pasada (autoaceptación); un sentido de crecimiento continuo y desarrollo como persona (crecimiento personal); la creencia de que la propia vida tiene un propósito y un significado (propósito en la vida); el desarrollo y mantenimiento de relaciones de calidad y confianza con otros (relaciones positivas con otros); la capacidad de dirigir efectivamente la propia vida y el ambiente para satisfacer necesidades y deseos (dominio ambiental); y un sentido de autodeterminación personal (autonomía).

El bienestar se encuentra influido por diferentes características sociodemográficas como edad, género y estado civil, pero sin duda un predictor importante del bienestar es la evaluación subjetiva que realiza el individuo de sus recursos, entre los cuales se encuentra el apoyo social percibido (Hicks, Epperly \& Barnes, 2001).

El apoyo social puede ser conceptualizado a partir de dos perspectivas, una cuantitativa-estructural y otra cualitativa-funcional. La primera evalúa la cantidad de vínculos o relaciones que establece el individuo con su red social, que correspondería al apoyo social recibido. En tanto la segunda se centra en la existencia de relaciones significativas y la evaluación o apreciación subjetiva que realiza el individuo de la adecuación del apoyo que recibe, correspondería al apoyo social percibido (Barra, 2004; Escobar, Puga \& Martín, 2008).

Entre los apoyos que recibe el adulto mayor, la familia es uno de los recursos que brindan mayor beneficio a la persona, al entregar atención y protección en los procesos físicos y mentales por los que atraviesan, en especial cuando la persona no posee el apoyo de redes formales (Domínguez, Espín \& Bayorre, 2001). Así, se ha encontrado que los adultos mayores que conviven con sus familiares presentan mayores niveles de salud mental, física y emocional en comparación con aquellos que viven solos (Bozo, Toksabay \& Kürüm, 2009). Dentro de la familia se ha observado que el matrimonio constituye un apoyo fundamental, especialmente para los hombres, ya que estos además de presentar una menor red social, dependen de sus esposas en la realización de actividades (Reyes, Camacho, Eschbach \& Markides, 2006).

El apoyo social es un elemento protector de la salud en los adultos mayores, al moderar los efectos negativos del estrés y contribuir así al bienestar y satisfacción con la vida (Aguerre \& Bouffard, 2008; Fernández, Clúa, Báez \& Ramírez, 2000). En el adulto mayor existe un aumento de las enfermedades crónicas y discapacidades que afectan la calidad de vida, incrementan las demandas de atención en los servicios de salud y el uso y consumo de fármacos (Hambleton, Clarke, Broome, Fraser, Brathwaite \& Hennis, 2005). Bajo la perspectiva biopsicosocial de la salud, el estilo y calidad de vida del adulto mayor estarán determinados por el contexto social en el que se encuentra inmerso, es decir, por la interacción entre las características del individuo y su entorno (Azpiazu, Jentoft, Villagrasa, Abanades, García \& Alvear, 2002; García \& González, 2000).

La percepción del adulto mayor acerca de su estado de salud y calidad de vida son influidos por su salud mental y capacidad funcional (Azpiazu et al., 2002; Love, Goldman \& Rodríguez, 2008). Por tanto, la percepción de salud es un constructo asociado a otras variables psicológicas como autoestima, satisfacción con la vida y depresión (Mella et al., 2004; Winocur, Palmer, Dawson, Binns, Bridges \& Stuss, 2007) y ha probado tener asociaciones significativas con otros indicadores más objetivos, como son el número de enfermedades crónicas que los adultos mayores padecen, el periodo de tiempo que han vivido con una enfermedad, la agudización de problemas crónicos, etc. (Beaman, Reyes, García-Peña \& Cortés, 2004).

En cuanto a posibles diferencias de género en el bienestar de los adultos mayores la evidencia no es consistente. Algunos estudios han encontrado que las mujeres reportan menores niveles de bienestar, mayores niveles de agotamiento y menor actividad física que los hombres (Alvarado, Zunzunegui, Béland \& Bamvita, 2008; Barrantes, 2006). Sin embargo, también hay estudios que han encontrado 
mayores niveles de bienestar en las mujeres, lo cual podría explicarse por su mayor estabilidad emocional con el aumento de la edad, y la comparación social que realiza la persona en la evaluación de las circunstancias objetivas que vive (Hicks et al., 2001; Pinguart \& Sorensen, 2001). Otra posible explicación sería que al envejecer los hombres se tornan más dependientes ante la pérdida del rol laboral que los define socialmente, en tanto las mujeres mantienen en mayor medida su rol social, al cuidar de su grupo familiar (Stefani, 2004).

Por último, el estado civil sería otra variable que puede influir en el bienestar en el contexto del envejecimiento. Pinguart y Sorensen (2001) señalan que los adultos mayores casados poseen un importante recurso de apoyo y validación de parte de su pareja, por lo cual las personas divorciadas serían más propensas a enfermar y presentan mayores índices de mortalidad y accidentes que aquellas casadas.

En base a la revisión de estudios previos, en este estudio se plantearon las siguientes hipótesis:

1. El bienestar psicológico de los adultos mayores se relacionará de forma significativa con el apoyo social percibido y con la percepción de salud.

2. Los adultos mayores de género masculino presentarán mayor nivel de bienestar psicológico.

3. Los adultos mayores casados o que tienen pareja presentarán mayor nivel de bienestar psicológico.

\section{Método}

\section{Participantes}

La muestra estuvo conformada por 250 personas, 146 mujeres y 104 hombres, con edades entre 60 y 87 años (promedio de 70,8 años), participantes de la Unión Comunal de Adultos Mayores de la comuna de Concepción.

\section{Instrumentos}

Encuesta sociodemográfica: cada participante completó una ficha acerca de datos demográficos generales (edad, estado civil, nivel educacional), lugar de residencia, enfermedades y autoevaluación del estado de salud con 4 alternativas, desde mala (1) hasta excelente (4).

Escalas de Bienestar Psicológico de Ryff (Díaz et al., 2006): compuestas por 39 ítems, con un formato de respuesta tipo Likert de cinco alternativas, desde "completamente de acuerdo" a "completamente en desacuerdo". Incluye seis escalas: auto-aceptación (6 ítems), relaciones positivas con otros (6 ítems), autonomía (8 ítems), dominio del entorno (6 ítems), propósito en la vida (6 ítems) y crecimiento personal ( 7 ítems). La consistencia interna de las escalas, mediante el coeficiente $\alpha$ de Cronbach varía entre 0.83 (auto-aceptación) y 0.68 (crecimiento personal).
Escala Multidimensional de Apoyo Social Percibido (MSPSS) de Zimet. (Arechabala \& Miranda, 2002): compuesta por 12 ítems que recogen información del apoyo social percibido por los individuos en tres áreas: familia, amigos y otros significativos. Posee una escala de respuesta tipo Likert de 4 puntos, desde "casi nunca" a "casi siempre". La confiabilidad por consistencia interna mediante el coeficiente $\alpha$ de Cronbach es de 0.86 .

\section{Procedimiento}

La recogida de datos se llevó a cabo durante reuniones de los distintos clubes de la Organización Comunal del Adulto Mayor de Concepción. Los instrumentos se aplicaron de manera colectiva y anónima, previa lectura y firma de una carta de consentimiento informado.

\section{Resultados}

Antes de proceder al análisis de los datos obtenidos se realizó un análisis de la confiabilidad de los instrumentos. En la Escala Multidimensional de Apoyo Social Percibido se obtuvo una alta consistencia interna mediante el coeficiente $\alpha$ de Cronbach de 0.92. En cambio en las Escalas de Bienestar Psicológico de Ryff se detectaron problemas de confiabilidad en dos escalas, lo que llevó a eliminar 3 de los 7 ítems de la escala de crecimiento personal y a eliminar por completo la escala de dominio del entorno compuesta por 6 ítems, ya que en ese caso la eliminación de algunos ítems no mejoraba su confiabilidad. Por lo tanto en los análisis que se describen a continuación, el bienestar psicológico global corresponde a la suma de los puntajes de las cinco escalas restantes (Autoaceptación, Relaciones positivas, Autonomía, Crecimiento personal y Propósito en la vida), con un total de 30 ítems.

En la Tabla 1 se puede apreciar que el bienestar psicológico global presenta una correlación moderada con la percepción de salud, relación que es notoriamente mayor en los hombres que en las mujeres, y por otra parte presenta una relación muy significativa con el apoyo social percibido, de una magnitud similar en ambos sexos.

Respecto a las distintas dimensiones del bienestar psicológico, la percepción de salud alcanza su mayor relación, de nivel moderado, con la dimensión de autoaceptación (especialmente en los hombres). En cambio el apoyo social percibido presenta relaciones muy significativas en ambos sexos con tres dimensiones del bienestar psicológico: relaciones positivas, autoaceptación y propósito en la vida. Por otro lado, la dimensión de autonomía muestra la menor relación con el apoyo social percibido en la muestra total y particularmente en las mujeres.

Para examinar las diferencias de género en las diversas variables se realizó un análisis de prueba t de diferencia de medias para muestras independientes, cuyos resultados 
Tabla 1. Correlaciones entre las diferentes variables en la muestra total y en cada sexo.

\begin{tabular}{|c|c|c|c|c|c|c|}
\hline \multirow[b]{2}{*}{ Variables } & \multicolumn{2}{|c|}{ Total } & \multicolumn{2}{|c|}{ Hombres } & \multicolumn{2}{|c|}{ Mujeres } \\
\hline & Perc. salud & Apoyo social & Perc. salud & Apoyo social & Perc. salud & Apoyo social \\
\hline Bienestar Psicológico global & $0.24 * *$ & $0.52 * *$ & $0.28 * *$ & $0.51 * *$ & $0.14 *$ & $0.54 * *$ \\
\hline Autoaceptación & $0.24 * *$ & $0.48 * *$ & $0.30 * *$ & $0.46^{* *}$ & $0.16^{*}$ & $0.49 * *$ \\
\hline Propósito en la vida & $0.19 * *$ & $0.46^{* *}$ & $0.25 * *$ & $0.53 * *$ & 0.13 & $0.41 * *$ \\
\hline Relaciones positivas con otros & $0.16^{* *}$ & $0.51 * *$ & $0.26^{* *}$ & $0.52 * *$ & 0.06 & $0.50 * *$ \\
\hline Crecimiento personal & $0.16^{* *}$ & $0.27 * *$ & $0.17 *$ & $0.20^{*}$ & $0.15^{*}$ & $0.31 * *$ \\
\hline Autonomía & $0.11^{*}$ & $0.14^{*}$ & 0.12 & $0.23 * *$ & 0.03 & 0.08 \\
\hline
\end{tabular}

$* \mathrm{p}<0.05 * * \mathrm{p}<0.01$

Tabla 2. Diferencias de género en las variables

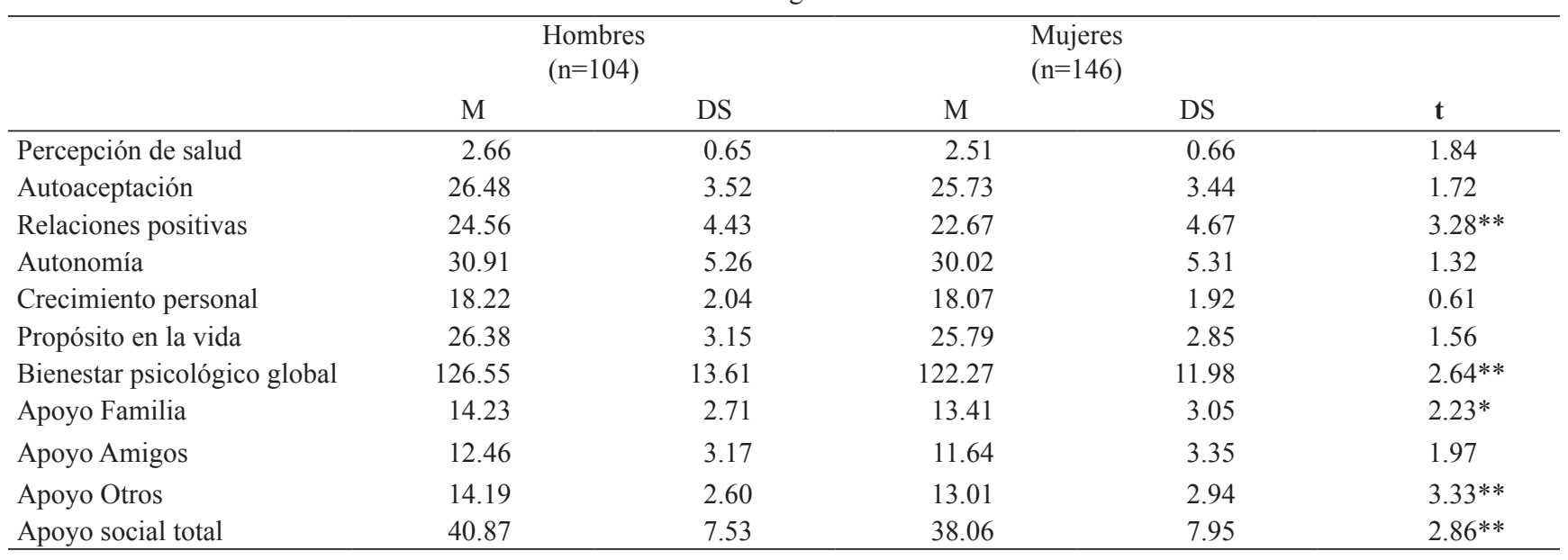

$* \mathrm{p}<0.05 * * \mathrm{p}<0.01$

Tabla 3. Diferencias en las variables entre personas con y sin pareja

\begin{tabular}{|c|c|c|c|c|c|}
\hline & \multicolumn{2}{|c|}{$\begin{array}{c}\text { Con pareja } \\
(\mathrm{n}=118)\end{array}$} & \multicolumn{2}{|c|}{$\begin{array}{c}\text { Sin pareja } \\
(\mathrm{n}=132)\end{array}$} & \multirow[b]{2}{*}{$\mathbf{t}$} \\
\hline & M & DS & M & DS & \\
\hline Percepción de salud & 2.66 & 0.65 & 2.51 & 0.66 & 1.84 \\
\hline Autoaceptación & 26.48 & 3.52 & 25.73 & 3.44 & 1.72 \\
\hline Relaciones positivas & 24.56 & 4.43 & 22.67 & 4.67 & $3.28 * *$ \\
\hline Autonomía & 30.91 & 5.26 & 30.02 & 5.31 & 1.32 \\
\hline Crecimiento personal & 18.22 & 2.04 & 18.07 & 1.92 & 0.61 \\
\hline Propósito en la vida & 26.38 & 3.15 & 25.79 & 2.85 & 1.56 \\
\hline Bienestar psicológico global & 126.55 & 13.61 & 122.27 & 11.98 & $2.64 * *$ \\
\hline Apoyo Familia & 14.23 & 2.71 & 13.41 & 3.05 & $2.23 *$ \\
\hline Apoyo Amigos & 12.46 & 3.17 & 11.64 & 3.35 & 1.97 \\
\hline Apoyo Otros & 14.19 & 2.60 & 13.01 & 2.94 & $3.33 * *$ \\
\hline Apoyo social total & 40.87 & 7.53 & 38.06 & 7.95 & $2.86^{* *}$ \\
\hline
\end{tabular}

$* \mathrm{p}<0.05 * * \mathrm{p}<0.01$ 
se presentan en la Tabla 2. Se puede observar que existen diferencias de género significativas, a favor de los hombres, en las variables percepción de salud y bienestar psicológico global, no observándose diferencias entre hombres y mujeres en la percepción de apoyo social.

Respecto a las dimensiones de bienestar psicológico, los hombres obtienen mayor puntaje que las mujeres en todas las dimensiones, con la excepción de crecimiento personal. Se destaca como la diferencia de género de mayor magnitud la referida a la dimensión autonomía.

Para analizar posibles diferencias en las variables entre las personas con y sin una pareja estable, se categorizó a los participantes en dos grupos, uno de ellos conformado por las personas casadas y convivientes, y el otro grupo conformado por las personas solteras, separadas y viudas.

La comparación de ambos grupos mediante la prueba $t$ de diferencia de medias (Tabla 3 ) muestra que las personas con pareja (casadas y convivientes) informan mayor bienestar psicológico global y mayor percepción de apoyo social que las personas sin pareja (solteras, separadas y viudas). Respecto a las dimensiones de bienestar psicológico, la única diferencia que alcanza significación estadística es la referente a relaciones positivas con otros. También se observa que las personas con pareja informan mejor percepción de salud que aquellas sin pareja, aunque tal diferencia no alcanza a ser significativa $(p=0.06)$.

\section{Discusión}

Los resultados obtenidos permitieron confirmar las hipótesis planteadas, mostrando la relevancia que tienen las variables percepción de salud, apoyo social percibido, estado civil y género para el bienestar psicológico de los adultos mayores.

Respecto a la primera hipótesis, tanto el apoyo social percibido como la percepción de salud presentaron una relación significativa con el bienestar psicológico, aunque la primera variable tiene una relación notoriamente mayor que la segunda con el bienestar psicológico de los adultos mayores. Este resultado es consistente con diversos estudios que destacan la importancia del apoyo que percibe el individuo de su entorno como elemento protector en la salud mental, ya que la existencia de relaciones significativas y estables en el tiempo inciden en el autoconcepto, el nivel de funcionamiento y el afrontamiento del estrés (Aguerre \& Bouffard, 2008; Barra, 2004; Fernández et al., 2000; Fiori, Smith \& Antonucci, 2007; Hicks et al., 2001; Kleinspehn, Kotte. \& Smith, 2008). Existen evidencias de que la existencia de una red social adecuada podría influir en la modificación de las estrategias de afrontamiento, en la integración o participación social y en el incremento de la motivación, actuando como un posible predictor de discapacidad en la vejez (Escobar et al., 2008; Mella et al., 2004;
Stefani, 2004). El apoyo social percibido no solo influiría en el bienestar al proporcionar estabilidad emocional, atención y protección de otros (Bozo et al., 2009; Domínguez et al., 2001), sino que también permitiría al individuo un mayor control percibido, sentirse productivo y tener un sentido en la vida (Azpiazu et al., 2002).

La relación entre percepción de salud y bienestar psicológico puede fundamentarse en que la salud constituye un estado global de bienestar y no simplemente de ausencia de enfermedades. El percibirse como una persona funcional, capaz de desenvolverse, se asocia a una mejor calidad de vida (Mella et al., 2004; Love et al., 2008), dentro de la cual la dimensión emocional tiene una relación directa con la salud, tanto en sus aspectos objetivos (morbilidad) como subjetivos (percepción de salud), en una relación recíproca. Por una parte, la salud influye sobre el bienestar de las personas mayores y por otra parte las actitudes positivas tienen un efecto protector sobre la salud y la calidad de vida (Mella et al., 2004; Prieto et al., 2008). La relación entre percepción de salud y bienestar psicológico en esta muestra es de un nivel moderado y muy inferior a la relación entre bienestar y apoyo social, lo cual se podría explicar por el hecho de que los adultos mayores participantes tenían en general un buen estado de salud y todos ellos se mantienen físicamente activos.

En cuanto a la segunda hipótesis, los hombres informaron mayor bienestar psicológico que las mujeres, tanto a nivel global como en la mayoría de sus dimensiones. Estos resultados coinciden con otras investigaciones que establecen factores del ciclo vital-social específicos al género, por los cuales las mujeres se encuentran en una desventaja y en algunos casos en una mayor vulnerabilidad (Alvarado et al., 2008). Es importante destacar que esta diferencia de género aparece muy significativa si se considera que las mujeres de la muestra participan en talleres y potencialmente poseerían recursos externos adicionales en relación a aquellas que no lo hacen, como sentirse más participes de la vida social (Chen \& Chan, 2006; Rioseco et al., 2008). Por tanto, la asociación entre género, vejez y bienestar sería compleja, compuesta por varias dimensiones, asociada también a condiciones de vida como si requiere asistencia especializada o se encuentra en una dependencia económica.

A diferencia de lo que podría esperarse, no existen diferencias significativas entre hombres y mujeres en apoyo social percibido. Una posibilidad explicativa sería que la percepción de apoyo social estaría influida no sólo por variables de género sino que por otras variables personales, por ejemplo la apreciación subjetiva de sus recursos propios de afrontamiento (Chen \& Chan, 2006; Mella et al., 2004). Otra alternativa es que la muestra se encuentra constituida por personas que comparten la misma red social, siendo relevantes para ellos los talleres a los que asisten en la Organización Comunal del Adulto Mayor, al sentirse 
partícipes de la sociedad y mantenerse activos (Rioseco et al., 2008).

En lo referente a la tercera hipótesis, los adultos mayores casados o que tienen pareja informan mayor nivel de bienestar psicológico global que los que no se encuentran en pareja. Esta diferencia puede explicarse por el de hecho que las personas que se encuentran sin pareja estable (separados o divorciados) presentarían más dificultades para adaptarse física y psicológicamente, porque también presentan menor apoyo social percibido, el cual sería un importante recurso de afrontamiento del estrés psicosocial. Al examinar las dimensiones del bienestar psicológico, se observa que existen diferencias significativas entre ambos grupos sólo en la dimensión de relaciones positivas con otros, lo cual apoyaría la interpretación anterior, en el sentido de que sería el apoyo social percibido el que explicaría en gran medida la diferencia en bienestar psicológico global entre ambos grupos. Es decir, la variable estado civil no afectaría por sí misma el bienestar psicológico del adulto mayor, siendo mediada esa relación por la evaluación subjetiva de los propios recursos personales y del entorno (Fernández, Almuniña, Alonso \& Blanco, 2001).

Los resultados obtenidos revelan la importancia de Programas Comunitarios en que se incentive la formación de redes sociales y el fomento del apoyo social, al ser factores protectores para el bienestar psicológico, permitiendo el desarrollo funcional de los adultos mayores, mediante el incremento de sentimientos de pertenencia e identidad. Asimismo sería importante trabajar en programas de promoción y autocuidado, destacando las actividades grupales que permitan el apoyo y compañía entre los adultos mayores. Todo esto en el contexto actual en que existe una tendencia al descenso en la fecundidad, el aumento en la expectativa de vida y el incremento en la proporción de este grupo etario (Mella et al., 2004). En este sentido, el mantenerse activo y funcional permitirá que el adulto mayor se sienta útil y valorizado por su medio. Por lo cual se requerirá aumentar y diversificar los servicios sociales y de salud para la tercera edad, donde se debe considerar este nuevo perfil de adulto mayor que requiere nuevas formas de atención, y tener en cuenta las variables estudiadas con el propósito de integrar a la sociedad a este sector que sigue en aumento.

Para próximas investigaciones se debería continuar investigando la influencia de dichas variables comparando con adultos mayores que requieran asistencia especial, como por ejemplo aquellos que residan en Hogares o Asilos. Así como integrar variables como estrategias de afrontamiento, dada su incidencia en la evaluación subjetiva que realiza el individuo de sus recursos y de su entorno. También se debería investigar en otras poblaciones incorporando variables biomédicas, por ejemplo sintomatología, condiciones diagnosticadas, utilización de servicios de salud, medidas de incapacidad, etc. (Barrantes, 2006).
Por último, en futuros estudios sería importante diferenciar por edades dentro de este grupo etario, ya que no solo existe la tercera edad, sino que en la actualidad se habla de una cuarta edad, de acuerdo a la funcionalidad del adulto mayor.

\section{Referencias}

Aguerre, C., \& Bouffard, L. (2008). Envejecimiento exitoso: Teorías, investigaciones y aplicaciones clínicas. Revista de la Asociación Colombiana de Gerontología y Geriatría, 22, 1146-1162.

Alvarado, B., Zunzunegui, M., Béland, F., \& Bamvita, J. (2008). Life course social and health conditions linked to frailty in Latin American older men and women. Journals of Gerontology: Medical Sciences, 63, 1399-1406.

Arechabala, M., \& Miranda, C. (2002). Validación de una escala de apoyo social percibido en un grupo de adultos mayores adscritos a un programa de hipertensión de la Región Metropolitana. Ciencia y Enfermería, 8, 49-55.

Azpiazu, M., Jentoft, A., Villagrasa, J., Abanades, J., García, N., \& Alvear, F. (2002). Factores asociados a mal estado de salud percibido o mala calidad de vida en personas mayores de 65 años. Revista Española de Salud Publica, 76, 683-699.

Barra, E. (2004). Apoyo social, estrés y salud. Psicología y Salud, 14, 237-243.

Barrantes, M. (2006). Género, vejez y salud. Acta Bioethica, 12, 193-197. Beaman, P., Reyes, S., García, C., \& Cortés, A. (2004). Percepción de la salud entre los adultos mayores derechohabientes del Instituto Mexicano del Seguro Social. En O. Muñoz, C. García, \& L. Durán (Eds.), La salud del adulto mayor (pp. 117-138). México: Instituto Mexicano del Seguro Social. Recuperado el 10 de septiembre de 2009 desde: http:// bibliotecas.salud.gob.mx/gsdl/collect/publin1/index/assoc/HASHd0ef. dir/doc.pdf

Boone, J., \& Wink, P. (2006).The Third Age: A rationale for research. Annual Review of Gerontology \& Geriatrics, 26, 19- 34.

Bozo, O., Toksabay, N., \& Kürüm, O. (2009). Activities of daily living, depression, and social support among elderly Turkish people. Journal of Psychology, 143, 193-205.

Chen, S., \& Chan, A. (2006). Relationship with others and life satisfaction in later life: Do gender and widowhood make a difference? Journal of Gerontology, 61, 46-53.

Díaz, D., Rodríguez, R., Blanco, A., Moreno, B., Gallardo, I., \& Valle, C. (2006). Adaptación española de las escalas de bienestar psicológico de Ryff. Psicothema, 18, 572-577.

Domínguez, T., Espín, A., \& Bayorre, H. (2001). Caracterización de las relaciones familiares del anciano. Revista Cubana de Medicina General Integral, 17, 418-422.

Escobar, M. A., Puga, D., \& Martín, M. (2008). Asociaciones entre la red social y la discapacidad al comienzo de la vejez en las ciudades de Madrid y Barcelona en 2005. Revista Española de Salud Pública, $82,637-651$.

Fernández, N., Clúa, A., Báez, R., \& Ramírez, M. (2000). Estilos de vida, bienestar subjetivo y salud de los ancianos. Revista Cubana de Medicina General, 16, 6-12.

Fernández, S., Almuniña, M., Alonso, O., \& Blanco, B. (2001). Factores psicosociales presentes en la tercera edad. Revista Cubana de Higiene y Epidemiología, 39, 77-81.

Fiori, K., Smith, J., \& Antonucci, T. (2007). Social network types among older adults: A multidimensional approach. Journal of Gerontology, $62,322-330$.

García, V., \& González, I. (2000). La categoría bienestar psicológico: Su relación con otras categorías sociales. Revista Cubana de Medicina General Integral, 16, 586-592.

Hambleton, I., Clarke, K., Broome, H., Fraser, H., Brathwaite, F., \& Hennis, A. (2005). Historical and current predictors of self-reported health 
status among elderly persons in Barbados. Revista Panamericana de Salud Pública, 17, 342-352.

Hicks, J., Epperly, L., \& Barnes, K. (2001). Gender, emotional support, and well-being among the rural elderly. Sex Roles, 45, 15-30.

Kleinspehn, A., Kotter, D., \& Smith, J. (2008). Self-perceptions of aging: Do subjective age and satisfaction with aging change during old age? Journal of Gerontology, 63, 377-385.

Love, A., Goldman, N., \& Rodríguez, G. (2008). Is positive well-being protective of mobility limitations among older adults? Journal of Gerontology, 63, 321-327.

Mella, R., González, L., D’ Appolonio, J., Maldonado, I., Fuenzalida, A., \& Díaz, A. (2004). Factores asociados al bienestar subjetivo en el adulto mayor. Psykhe, 13, 79-89.

Molina, C., \& Meléndez, J. (2006). Bienestar psicológico en envejecientes de la República Dominicana. Geriátrika, 22, 97-105.

Pinguart, M., \& Sorensen, S. (2001).Gender differences in self-concept and psychological well-being in old age: A meta-analysis. Journal of Gerontology, 56, 195-214.

Prieto, M. E., Fernández, G., Rojo, F., Lardiés, R., Rodríguez, V., \& Ahmed, K. (2008). Factores sociodemográficos y de salud en el bienestar emocional como dominio de calidad de vida de las personas mayores en la Comunidad de Madrid: 2005. Revista Española de Salud Pública, 82, 301-313.
Reyes, C., Camacho, M., Eschbach, K., \& Markides, K. (2006). El contexto de la familia y el vecindario en la salud de los ancianos del estudio EPESE hispano. Colombia Médica, 37, 45-49.

Ryff, C., \& Singer, B. (2008). Know thyself and become what you are: A eudaimonic approach to psychological well-being. Journal of Happiness Studies, 9, 13-39.

Rioseco, R., Quezada, M., Ducci, M., \& Torres, M. (2008). Cambio en las redes sociales de adultos mayores beneficiarios de programas de vivienda social en Chile. Revista Panamericana de Salud Pública, $23,147-153$.

Sanhueza, M., Castro, M., \& Merino, J. (2005). Adultos mayores funcionales: Un nuevo concepto en salud. Ciencia y Enfermería, 11, 17-21.

Stefani, D. (2004). Gender and coping in old age. Interdisciplinaria, Revista de Psicología y Ciencias Afines. Recuperado en agosto 07 de 2009, disponible en http://redalyc.uaemex.mx/src/inicio/ArtPdfRed. jsp?iCve $=18009908$

Tomás, M., Meléndez, J., \& Navarro, E. (2008). Modelos factoriales confirmatorios de las escalas de Ryff en una muestra de personas mayores. Psicothema, 20, 304-310.

Winocur, G., Palmer, H., Dawson, D., Binns, M., Bridges, K., \& Stuss, D. (2007). Cognitive rehabilitation in the elderly: An evaluation of psychosocial factors. Journal of the International Neuropsychological Society, 13, 153-165. 